\title{
Relation between defect depth and standard thermal contrast on the steel surface in pulsed thermography
}

\author{
by M. Maj ${ }^{*}$ W. Oliferuk ${ }^{* *}$ and O. Wysocka*
}

\author{
*Institute of Fundamental Technological Research, Polish Academy of Sciences, Świętokrzyska 21, 00-049 \\ Warsaw, Poland. \\ ** Institute of Fundamental Technological Research, Polish Academy of Sciences and Białystok Technical \\ University, Wiejska 45A, 15-351 Białystok, Poland
}

\begin{abstract}
The work is devoted to experimental determination of the diameter and the depth of simulated subsurface defects in steel using pulse thermography (PT). The simulated defects had a form of flat-bottom holes of diameter 1.0-5.0 mm and depth $0.3-1.5 \mathrm{~mm}$. The defect diameter has been reconstructed on the basic of analysis of the rate of temperature decrease on the surface of tested specimen after its heating by the heat pulse. Defect depth has been estimated from the dependence of thermal contrast on the tested surface vs. self-cooling time. Experimental results are presented.
\end{abstract}

\section{Introduction}

Pulsed thermography (PT) is a non-destructive testing method that is based on the thermal excitation of the tested specimen and observation of self-cooling process of its surface by Infrared Imaging System. It is one of a few kinds of the active thermography [1-3]. This testing method has got unique features like: contact-free operation, possibility to inspect large area simultaneously and high inspection speed. The method is used for detection of subsurface defects such as delamination and inclusions having different thermal properties with respect to that of the sound material. The inspection usually includes three stages: thermal excitation of tested specimen, monitoring its temperature field on the surface after the excitation and data analysis. Nowadays, detection of presence subsurface defects using active thermography is rather easy.

The defect presence is usually manifested by an increase in the surface temperature over defected zone at the beginning of the self-cooling process after thermal excitation. Quantitative measure of a difference between surface temperature over the defect and over the sound material is for example standard thermal contrast [2]:

$$
C(t)=\frac{T_{\text {def }}(t)-T_{d}\left(t_{0}\right)}{T_{S}(t)-T_{S}\left(t_{0}\right)},
$$

where $T_{d e f}$ is the surface temperature over defect, $T_{S}$ is the temperature over the sound material, $t_{0}$ is the time before heating and $t$ is the current time of the process.

The results of analysis of thermal contrast vs. self-cooling time relation show that this dependence has got a maximum and the time related to the maximum is depended on both size of defect and its depth [4]. As it is seen in Fig. 1, the time of the standard thermal contrast maximum can be the same for flat-bottom holes with different depths and different diameters. That means that in order to determine the defect depth on the basis on thermal contrast analysis it is necessary to know the size of the defect.

The purpose of this work is to find the depth of the defects from the thermal contrast analysis taking into account reconstructed values of defect diameters. In this work the diameter of the flat-bottom holes was experimentally reconstructed on the basis of analysis of the rate of temperature decrease.

\section{Experimental procedure and results}

The experiments were performed on the specimen made of $316 \mathrm{~L}$ austenitic stainless steel plate $(250 \mathrm{~mm} x$ $170 \mathrm{~mm} \times 3 \mathrm{~mm}$ ) with flat-bottom hole defects. The diameters of defects $d$ are 1, 2, 3, 4, $5 \mathrm{~mm}$ of the depths 0.3 , $0.5,0.7,0.9,1.2$ and $1.5 \mathrm{~mm}$ (Fig. 2). The surface of specimen was coated by graphite paint and uniformly heated using the lamp of the pulse energy of $5 \mathrm{~kJ}$. Pulse duration was $3 \mathrm{~ms}$ and the lamp to specimen distance was 0.5 $\mathrm{m}$. Infrared imaging system allows to record temperature distribution on the tested surface vs. self-cooling time. Both the IR camera and stimulating lamp were located at the same side of the specimen (see Fig. 3). The surface temperature distribution was recorded with frame rate $346 \mathrm{~Hz}$. The full frame size was 320x256 pixels.

The subsurface defects can be detected mainly because of different rate of self-cooling of particular areas of the tested surface due to difference between thermal diffusivity of defects and of tested material. Thus, the surface distribution of self-cooling rate seems to be better for detection of subsurface defects than simple surface 
temperature distribution. As a measure describing surface self-cooling rate the time derivative of the temperature was used.

In order to obtain the time derivative of the temperature the experimentally found dependence surface temperature vs. self-cooling time was approximated using root model function for each pixel of thermal image. The results of such operation for the flat-bottom holes of the depth $0.3 \mathrm{~mm}$ at the time $78 \mathrm{~ms}$ after thermal excitation are presented in Fig. 4.

Analysis of relation temperature derivative vs. self-cooling time has shown that the dimensions of surface thermal contour of defects remain constant during some period of time. The results of analysis are consisted with that presented in [5]. This is time period when increase in standard thermal contrast is observed. It has been found that the dimensions of the thermal contour in this period are almost equal to real diameter of defects (see Table 1). Thus, the diameter of defects can be reconstructed from surface distribution of temperature time derivative. The values of thermal contour diameter presented in Table 1 were determined just after appearance of thermal contour related to particular defects. The higher increase of standard thermal contrast you have, the better accuracy of defect size reconstruction you get. Determination of the contour diameter is not possible if the increase in standard thermal contrast does not appear. Because of the insufficient rise in the standard thermal contrast it was impossible to reconstruct the diameter of some flat-bottom holes (see Table 1).

As mentioned above, the depth of the defect can be determined on the basis of the time thermal contrast dependence if the defect diameter is known. The standard thermal contrast as a function of self-cooling time was calculated for surface area over each defect. Taking into account reconstructed values of defect diameters, analysis of the dependence of standard thermal contrast (Eq. 1) vs. time was performed. The results of such analysis for flat-bottom hole of diameter $3 \mathrm{~mm}$ are presented in Fig. 5.

It is seen that the dependence of the standard thermal contrast vs. the self-cooling time has a maximum. It is characteristic that the time of the $C(t)$ maximum appearance is different for different depths of the defects. The smaller depth of defect corresponds to the shorter time to reach the maximum. From the standard thermal contrast vs. time dependence for different diameters of simulated defects calibration curves have been created. The calibration curve is a relation between defect depths vs. time of standard thermal contrast maximum for given diameter of flat-bottom hole. Calibration curves for flat-bottom holes are presented in Fig. 6 . It is seen that for every defects diameter this dependence is a straight line with the same slope.

Presented approach allows us to determine the defect diameter. After calibration process for given material the depth of defect can be assessed.

\section{Concluding remarks}

Surface distribution of the temperature derivative with respect to self-cooling time after thermal stimulation of the specimen was used for reconstruction of simulated defects diameter in subsurface layer of stainless steel.

The dependences of standard thermal contrast vs. self-cooling time for different size of defects and for different depths were experimentally determined. It has been shown that if the higher increase of standard thermal contrast is observed, the better accuracy of defect size reconstruction can be obtained.

Analysis of time standard thermal contrast evolution was performed and reconstructed diameters were used to determine the calibration curves. The calibration curve is the relation between defect depths and the time of standard thermal contrast maximum for given diameter of flat-bottom hole. Presented approach makes possible to find not only the diameter of real subsurface defect but also its depth for given material.

The further effort for our work is to perform such kind of analysis for defects of different shapes and orientations with respect to the tested surface.

\section{REFERENCES}

[1] V.P. Vavilov, T. Ahmed, H.J. Jin, L.R. Favro, L.D. Thomas, Experimental thermal tomography of solids by using the pulse one-side heating, Soviet Journal of Nondestructive Testing, 12 (1990) 60-66.

[2] X. Maldaque. Theory and practice of infrared technology for non-destructive testing. Willey (2001)

[3] S. Lugin, U. Netzelmann. A defect shape reconstruction algorithm for pulse thermography, NDT\&E International, 40 (2007) 220-228.

[4] W. Oliferuk, Z. Płochocki, O. Wysocka. Pulse thermography with finie duration of stimulating heat impulse, (2008) submitted to NDT\&E.

[5] A.R. Hanzah, P. Delpech, M.B. Saintey, D.P. Almond. The experimental investigation of defect sizing by transient thermography, Insight, 38, 3 (1996) 167-171. 
Table 1. Comparison of flat-bottom holes diameter with reconstructed one.

\begin{tabular}{|c|c|c|}
\hline $\begin{array}{l}\text { defect depth } \\
{[\mathrm{mm}]}\end{array}$ & $\begin{array}{c}\text { reconstructed diameter } \\
{[\mathrm{mm}]}\end{array}$ & $\begin{array}{c}\text { flat-bottom hole diameter } \\
{[\mathrm{mm}]}\end{array}$ \\
\hline 0,3 & 4,87 & \multirow{6}{*}{4,95} \\
\hline 0,5 & 5,00 & \\
\hline 0,7 & 4,99 & \\
\hline 0,9 & 5,15 & \\
\hline 1,2 & 5,14 & \\
\hline 1,5 & - & \\
\hline 0,3 & 3,99 & \multirow{6}{*}{3,96} \\
\hline 0,5 & 3,98 & \\
\hline 0,7 & 4,19 & \\
\hline 0,9 & 4,23 & \\
\hline 1,2 & - & \\
\hline 1,5 & - & \\
\hline 0,3 & 2,98 & \multirow{6}{*}{2,91} \\
\hline 0,5 & 2,99 & \\
\hline 0,7 & 3,29 & \\
\hline 0,9 & - & \\
\hline 1,2 & - & \\
\hline 1,5 & - & \\
\hline 0,3 & 2,03 & \multirow{6}{*}{1,97} \\
\hline 0,5 & 1,98 & \\
\hline 0,7 & 2,08 & \\
\hline 0,9 & - & \\
\hline 1,2 & - & \\
\hline 1,5 & - & \\
\hline 0,3 & 0,95 & \multirow{6}{*}{0,96} \\
\hline 0,5 & 0,99 & \\
\hline 0,7 & - & \\
\hline 0,9 & - & \\
\hline 1,2 & - & \\
\hline 1,5 & - & \\
\hline
\end{tabular}

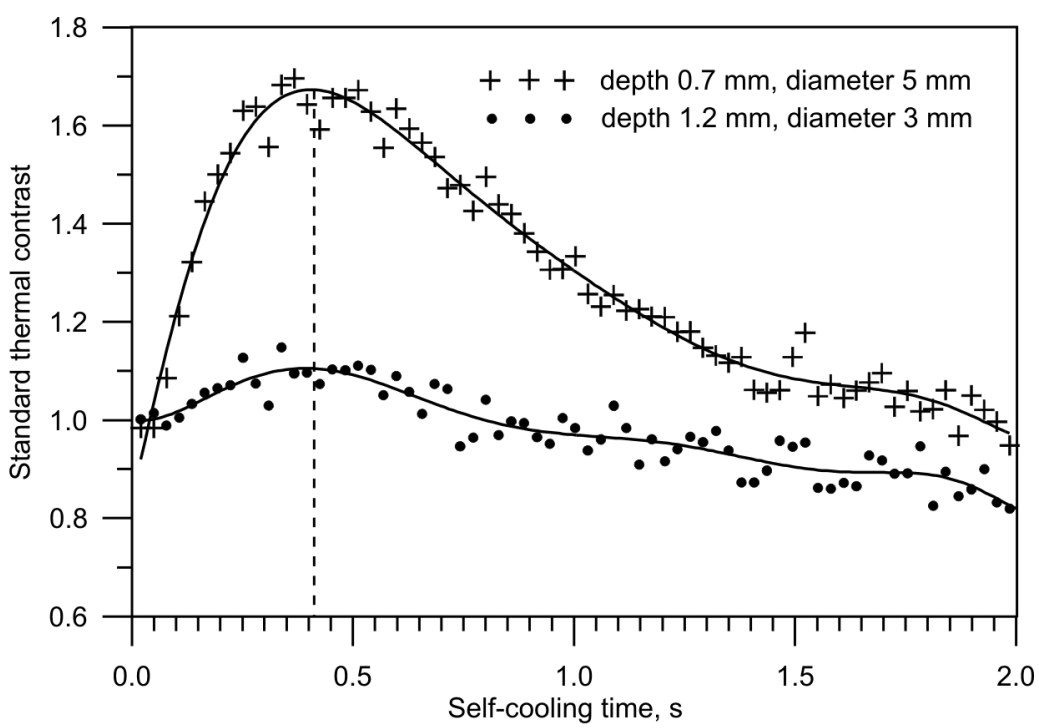

Fig. 1. The time of standard thermal contrast maximum is the same for flat-bottom holes of different depths are 0.7 and $1.2 \mathrm{~mm}$. Defect diameters are 5 and $3 \mathrm{~mm}$, respectively. 


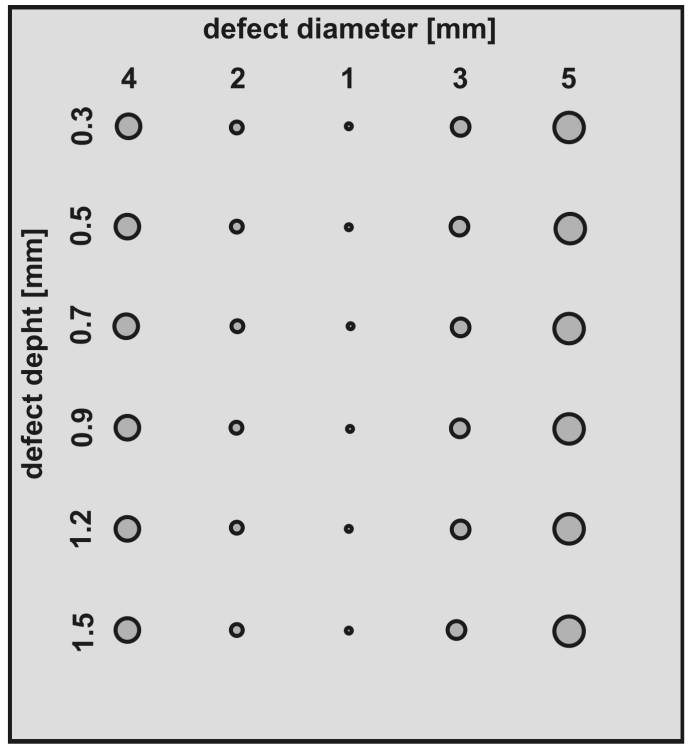

Fig. 2. The geometry of the steel plate with simulated defects (flat-bottom holes).

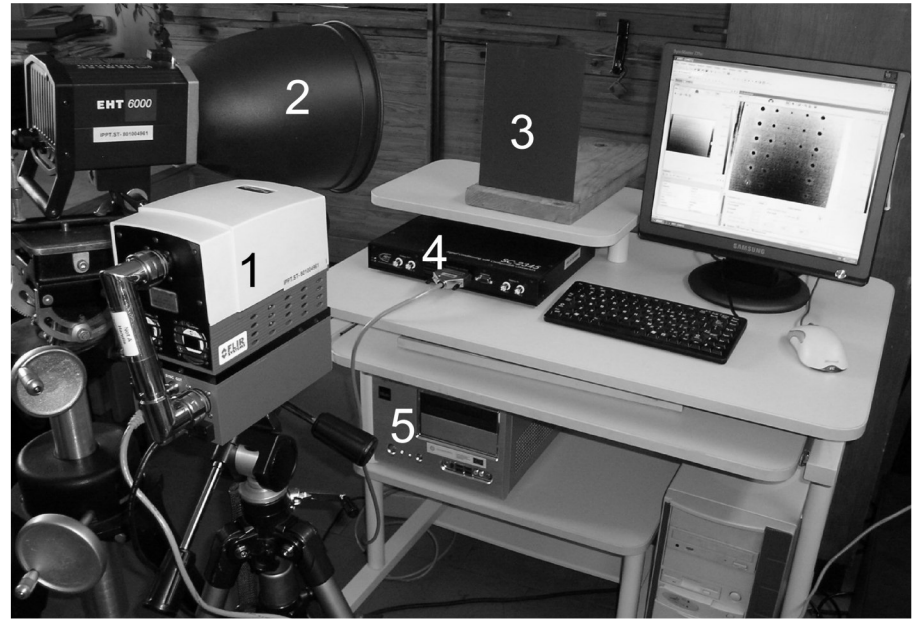

Fig. 3. Experimental set-up for pulse thermography: 1 - IR camera, 2 - flash lamp, 3 - tested specimen, 4 interface between IR camera and excitation lamp, 5 - computer.

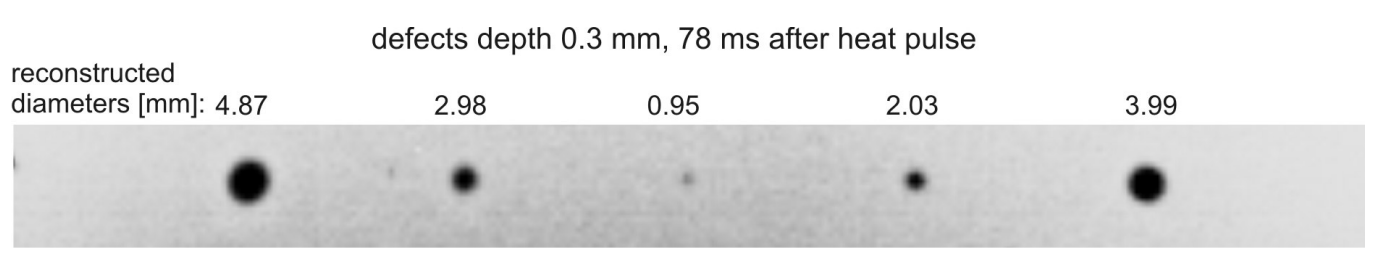

Fig. 4. Surface distribution of the time derivative of temperature (defects depth $0.3 \mathrm{~mm}$, self-cooling time $78 \mathrm{~ms}$ ). 


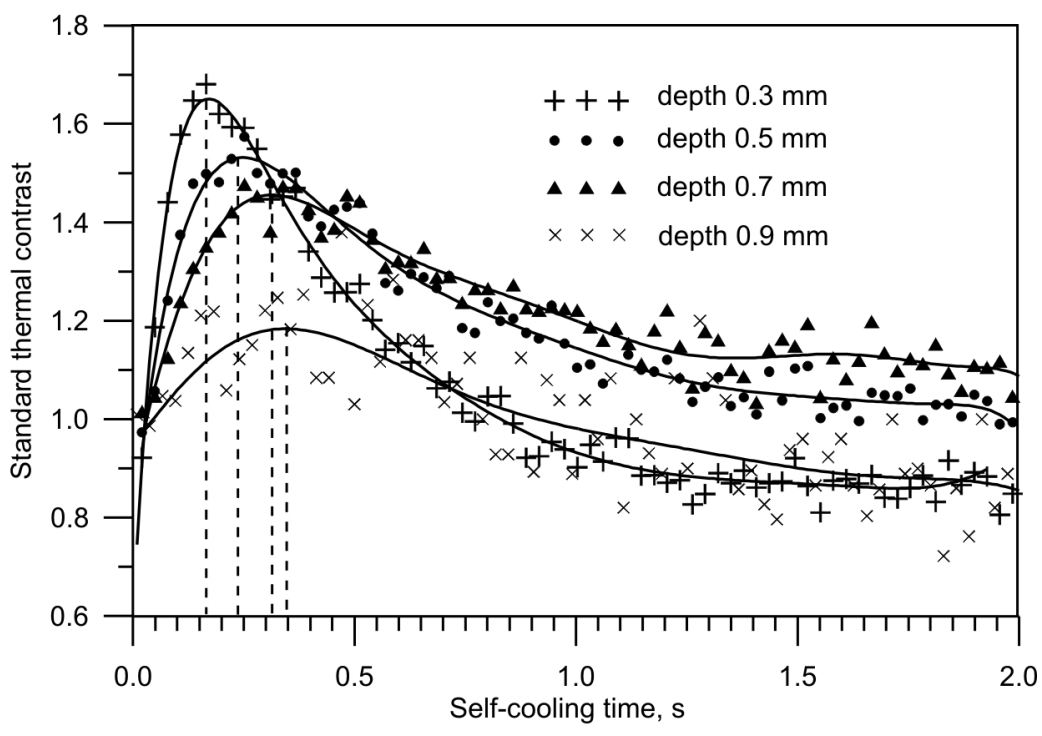

Fig. 5. Time evolution of the standard thermal contrast. Defect diameter $d=3 \mathrm{~mm}$. The time of standard thermal contrast maximum is marked.

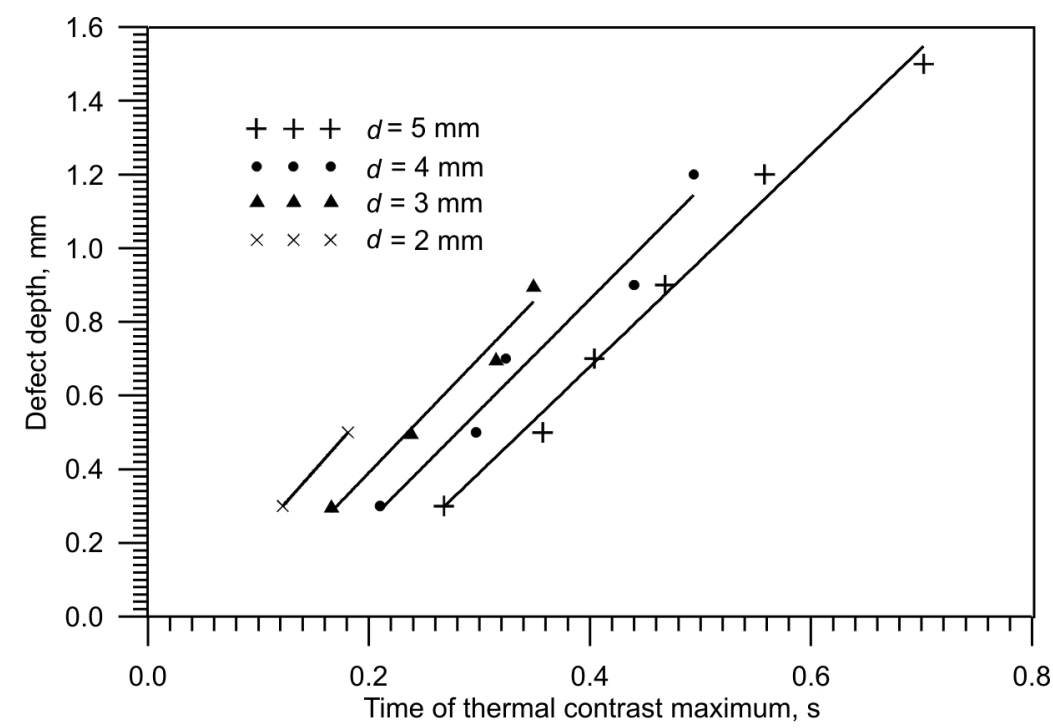

Fig. 6. Dependence of depth vs. time of thermal contrast maximum (the calibration curves). 
http://dx.doi.org/10.21611/qirt.2008.15_12_12 